\title{
Large-Scale Analysis of Circulating Amino Acids and Adipose Gene Expression in Relation to Abdominal Obesity
}

Running Head: Amino acids and abdominal obesity

Maltais-Payette Ina ${ }^{1,2}$, Vijay Jinchu ${ }^{3,4}$, Simon Marie-Michelle ${ }^{4}$, Jacques Corbeil ${ }^{5,6}$, Francis

Brière $^{5,6}$, Grundberg Elin* ${ }^{7}$, Tchernof André*1,2

${ }^{1}$ Quebec Heart and Lung Institute, Laval University, Canada

${ }^{2}$ School of Nutrition, Laval University, Canada

${ }^{3}$ Department of Human Genetics, McGill University, Montreal, Québec, Canada.

${ }^{4}$ McGill University and Genome Quebec Innovation Centre, Canada

${ }^{5}$ Departement of Molecular Medicine, Laval University, Canada

${ }^{6}$ Laval University Hospital Center, Canada

${ }^{7}$ Genomic Medicine Center, Children's Mercy Research Institute, Children's Mercy Kansas City, MO, USA.

\section{*Co-corresponding Authors:}

Elin Grundberg, PhD

Genomic Medicine Center

Children's Mercy Research Institute

Children's Mercy Kansas City

2401 Gilham Road

Kansas City, MO 64108, USA

Tel: +1 816-731-7084

Email: egrundberg@cmh.edu

Andre Tchernof, Ph.D.

Quebec Heart and Lung Institute

2725 Chemin Sainte-Foy (Y-4212)

Québec, Qc, Canada, G1V 4G5

Tel: +1 418-656-8711

Email: andre.tchernof@criucpq.ulaval.ca

Keywords: Glutamate, abdominal obesity, adipose tissue 


\section{ABSTRACT}

2 Background: Circulating level of the amino acid glutamate is associated with central fat

3 accumulation, yet the pathophysiology of this relationship remains unknown. We aimed to: i)

4 refine and validate the association between circulating glutamate and abdominal obesity in a

5 large population-based twin cohort; and ii) investigate whether transcriptomic profiles in adipose

6 tissue could provide insight into biological mechanisms underlying the association. Methods:

7 First, in a cohort of 4,665 individuals from the TwinsUK resource, we identified individuals with

8 abdominal obesity and compared prevalence of the latter across circulating glutamate quintiles.

9 Second, we used transcriptomic signatures generated from adipose tissue, both subcutaneous and

10 visceral, to investigate associations with circulating glutamate levels. Results: Individuals in the

11 top circulating glutamate quintile had a 7-fold higher prevalence of abdominal obesity compared

12 to those in the bottom quintile. The adipose tissue transcriptomic analyses identified $G L U L$,

13 encoding Glutamate-Ammonia Ligase, as being associated with circulating glutamate and

14 abdominal obesity, with pronounced signatures in the visceral depot. Conclusion: Circulating

15 glutamate is positively associated with the prevalence of abdominal obesity which relates to

16 dysregulated GLUL expression specifically in visceral adipose tissue. 


\section{INTRODUCTION}

2 In the past decade, studies based on metabolomics have allowed investigators to establish that

3 obesity is associated not only with alterations in circulating carbohydrates and lipids, but also in

4 circulating amino acids (1). The most studied are branched-chain amino acids (BCAAs), namely

5 leucine, isoleucine and valine. Indeed, they have been repeatedly demonstrated to be positively

6 associated with obesity, insulin resistance and the long-term risk of type 2 diabetes (T2D) as well

7 as cardiovascular diseases (1).

8 In studies investigating central fat accumulation specifically, rather then overall adiposity,

9 another amino acid has stood out: glutamate. We have shown that, out of nearly 200 metabolites,

10 glutamate was the most strongly associated with visceral adipose tissue (VAT) accumulation in a

11 sample of 59 middle-aged women (2). Although glutamate is much less studied than the BCAAs,

12 studies that did measure it generally found that it is more strongly correlated with central fat

13 accumulation than the BCAAs themselves, as shown in the Framingham Heart Study Generation

143 cohort for waist circumference (WC) (3) and in a sample of 1,499 Japanese participants for

15 VAT area measured by computed tomography (4). In the TwinsUK cohort, Menni et al.

16 investigated whether the relationship between circulating metabolites and T2D or blood pressure

17 was mediated by trunk fat mass (5). They found that the strongest independent association with

18 trunk fat was seen for glutamate. Of note, the three BCAAs were not identified as being

19 independently associated with trunk fat in this analysis. However, this was not the central

20 analysis of the study and as such, those results were not further explored.

21 Considering the strength of the association between circulating glutamate and abdominal obesity

22 and its consistency across cohorts as well as measurement tools of body fat distribution, we

23 aimed to explore this relationship further. In this study, we first confirm the association between 
1 circulating glutamate and abdominal obesity. Second, we identify the adipose tissue transcripts

2 associated with circulating glutamate to uncover pathways that relate to this association.

\section{METHODS}

\section{$4 \quad$ Study population and samples}

5 We used participant of the TwinsUK resource (6) where metabolomic and metabolic phenotypes

6 were available for analysis. The vast majority (94\%) of participants were females, and average

7 body mass index (BMI), WC, total cholesterol, triglycerides and homeostatic model assessment

8 of insulin resistance (HOMA-IR) were within normal ranges (see Table 1). To obtain

9 measurements of body fat distribution, we used the dual-energy absorptiometry (DXA)-derived

10 measurement of trunk fat and calculated the percentage of fat in the trunk region. Because no

11 official threshold for abdominal obesity exists for this measurement, we determined one using a

12 WC threshold of $95 \mathrm{~cm}$ (7). From the linear regression between trunk fat (\%) and WC, we

13 identified $36.7 \%$ trunk fat as a threshold for abdominal obesity. According to this definition,

$1446 \%$ of the cohort $(n=2139)$ presented abdominal obesity. Participants from the TwinsUK cohort

15 all provided informed consent. Secondary analysis of the TwinsUK cohort was approved by the

16 ethics committee of Laval University.

17 We also identified a subgroup of monozygotic (MZ) twins discordant for abdominal obesity as

18 follows. First, we calculated the intra-pair difference in trunk fat (\%) between individuals of the

19 MZ twin pairs. Next, we ranked the pairs based on the intra-pair difference and selected those

20 among the highest $\left(5^{\text {th }}\right)$ percentile which were consequently considered as discordant for

21 abdominal adiposity, representing 54 pairs. Age was calculated at the time blood was drawn for 
1 metabolomic measurements, and because in some cases the twins were not tested on the same

2 day, there is a small, non-significant difference in mean age of the 2 subgroups.

3 A subset $(n=776)$ of the TwinsUK cohort was also part of the MuTHER project, where

4 subcutaneous adipose tissue samples were collected and the transcriptome was profiled as

5 described previously $(8,9)$. In all, transcriptomics, metabolomics and DXA measurements were

6 available for 689 of the 776 women. In this subgroup, $57 \%$ of women $(n=395)$ had abdominal

7 obesity. Their complete characteristics are presented in Table $\mathbf{1 .}$

8 Finally, to investigate the adipose tissue transcriptome and its variance by depot, we used a

9 sample of bariatric surgery patients from the Quebec Heart and Lung Institute (IUCPQ, Laval

10 University, Quebec City, Canada). Informed consent was obtained from each participant through

11 the management framework of the IUCPQ Obesity Biobank. The protocol was approved by the

12 Research Ethics Committee of IUCPQ (Protocol \# 21320). Subcutaneous adipose tissue (SAT)

13 and omental VAT samples from 43 individuals were included in this study with BMI > 35.5

$14 \mathrm{~kg} / \mathrm{m}^{2}$ and age from 29 to 65 years. The majority of the participants were females $(\mathrm{n}=22)$

15 (Supplemental Table 4).

16 Amino acid measurement

17 In the TwinsUK cohort (including the MuTHER sample), amino acid measurements were

18 extracted from untargeted metabolomic performed by Metabolon Inc. as described

19 elsewhere (10). Briefly, plasma and serum samples were analyzed by ultra-high-performance

20 liquid chromatography-tandem mass spectrometry and metabolites were identified by automated

21 comparison. Metabolite concentrations were divided by the median of the day and inverse-

22 normalized. 
1 For the sample of bariatric participants, all amino acids were quantified using a Water Acquity

2 UPLC system coupled to a Synapt G2-Si mass spectrometer (Waters) in tandem mode (LC-

3 MS/MS) using the EZ:faast amino acid sample testing kit (Phenomenex, 2003). Briefly, plasma

4 samples were mixed with an internal standard solution and amino acids were extracted using a

5 solid-phase support. Extracted amino acids were derivatized to increase stability before analysis

6 and purified using a two-phase liquid-liquid extraction. Samples were analyzed using the HPLC

7 column, method gradient, and multiple reaction monitoring (MRM) transitions provided by the

8 kit. Quantification was done using sample to internal standard ratios and a 5-point calibration

9 curve ranging from $20 \mathrm{nmol} / \mathrm{mL}$ to $200 \mathrm{nmol} / \mathrm{mL}$. Our focus was on glutamate, but we also

10 performed all analyses on BCAA levels for comparison purposes.

\section{RNA Sequencing (RNASeq) in bariatric samples}

12 In the bariatric surgery sample, adipose tissue was digested within 30 minutes of collection using

13 collagenase adapting the Robdell method (11). The stroma-vascular fraction (SVF) cells,

14 adipocytes and whole tissue were cryopreserved and stored in liquid nitrogen. From the frozen

15 samples, 0.5 to 3 million cells were re-suspended in $500 \mathrm{uL}$ TRIzol Reagent and total RNA was

16 extracted using the miRNeasy Mini Kit (Qiagen) according to the manufacturer's protocol. RNA

17 library preparations were carried out on $500 \square$ ng of RNA with RNA integrity number (RIN)>7

18 using the Illumina TruSeq Stranded Total RNA Sample preparation kit, according to the

19 manufacturer's protocol. Final libraries were analyzed on a Bioanalyzer and sequenced on the

20 Illumina HiSeq 2500. The sequenced reads were pair-ended and $100-125$ bp in length. 
Statistical analyses

2 We evaluated the association between circulating amino acid levels and trunk fat using mixed

3 linear regressions. The models were adjusted for age, sex, metabolomic batch and twin-pair

4 clustering. We calculated the odds ratio (OR) of presenting abdominal obesity and metabolic

5 syndrome (MetS) features according to circulating amino acid quintiles using mixed logistic

6 regressions adjusted for age, sex, metabolomic batch and twin-pair clustering. We evaluated the

7 potential influence of genetics on the association between amino acids and trunk fat by

8 conducting a co-twin case-control analysis in MZ twin pairs discordant for abdominal obesity.

9 We compared circulating amino acid levels between leaner and heavier (in terms of trunk fat)

10 twins of each pair using paired and two-tailed Student' t-test.

\section{Transcriptomic analysis contrasting with metabolite measurements in MuTHER}

12 We used the transcriptome profile of healthy female twins from the MuTHER dataset (8) to

13 study changes in gene expression pattern in accordance to circulating glutamate levels.

14 Subcutaneous adipose tissue was profiled using Illumina Human HT12 array as previously

15 described $(8,9)$. We identified the individuals with circulating glutamate in quintiles 1 and 5

16 who had gene expression data $(n=209)$. In order to find the differentially expressed genes, we

17 used linear mixed model and compared gene expression with the quintile adjusted for age, sex,

18 metabolomic batch and twin-pair clustering. Further, we expanded our analysis with the data

19 obtained from bariatric participants. Circulating glutamate levels were compared with RNASeq

20 data normalized using DeSeq2. We used linear model to identify varying gene expression

21 patterns against metabolite measurements adjusted for the batch effect. 
2 The sequencing reads were trimmed for quality (phred33 $\geq 30$ ) and length $(\mathrm{n} \geq 32$ ), and Illumina

3 adapters were clipped off using Trimmomatic v.0.35 (12). Filtered reads were then aligned to the

4 GRCh37 human reference genome using STAR v.2.5.3a (13). Raw read counts of genes were

5 obtained using htseq-count v.0.6.0 (14). Differential gene expression analysis was done using

6 DeSeq2 v.1.18.1 (15). Ingenuity Pathway Analysis (IPA: QIAGEN Inc.,

7 https://www.qiagenbioinformatics.com/products/ingenuitypathway-analysis) was used for

8 identifying enriched pathways. To identify VAT-specific SVF genes, we did differential gene

9 expression analysis between visceral-specific SVF and matched adipocyte RNA-Seq data,

10 identifying 9,023 SVF-specific genes ( $\log 2 \mathrm{FC}$ of $>=2$, Adj. $\mathrm{p}<=0.05)$. The genes were further

11 filtered by restricting to those upregulated in VAT SVF in comparisons with SAT SVF using a

12 nominal $\mathrm{p}$ value $\leq 0.05$ and it resulted in 1,820 genes (Supplemental Table 6) including 1,117

$13(61 \%)$ protein-coding genes.

\section{RESULTS}

15 Association between circulating amino acid concentrations and abdominal obesity

16 We first investigated the biomarker potential of glutamate for abdominal obesity in the TwinsUK

17 cohort (6). For this analysis, we considered individuals for whom metabolomic and body

18 composition data were available and taken within 6 months of each other, which corresponded to

19 4,665 individuals. We applied a linear mixed model linking circulating glutamate with

20 measurements of obesity and found a significant and positive association with trunk fat (\%)

$21(\mathrm{n}=4665, \beta=0.23, \mathrm{p}$-value=2.1e-70) and BMI $(\mathrm{n}=4596, \beta=0.27, \mathrm{p}$-value=3e-78). We also tested

22 the association between circulating glutamate and additional metabolic and T2D-related traits 
1 and found positive associations with total triglycerides $(n=4058, \beta=0.29, p$-value $=1.2 \mathrm{e}-62)$ and

2 the HOMA-IR index $(\mathrm{n}=3881, \beta=0.31$, $\mathrm{p}$-value=5.7e-38) and a negative association with high-

3 density-lipoprotein cholesterol (HDL-C) $(n=2855, \beta=-0.23$, $p$-value=2.1e-32). Associations with

4 these variables were all stronger for glutamate than for any of the three BCAAs (Supplemental

5 Table 1).

6 Next, we computed the odds of presenting with abdominal obesity in the $2^{\text {nd }}, 3^{\text {rd }}, 4^{\text {th }}$ and $5^{\text {th }}$

7 circulating glutamate quintiles compared to the $1^{\text {st }}$ with mixed logistic regression models. Results

8 are presented in Figure 1. The odds of presenting abdominal obesity were significantly higher

9 for the $3^{\text {rd }}, 4^{\text {th }}$ and $5^{\text {th }}$ quintiles. Participants in the $5^{\text {th }}$ glutamate quintile also had a greater

10 prevalence of most MetS features: high WC (OR: 9.78, 95\%CI: 4.65, 20.58), high triglycerides

11 (OR: 7.28, 95\%CI: 4.84, 10.94), low HDL-C (OR: 4.91, 95\%CI: 2.94, 8.21) and high fasting

12 glucose (OR: $4.27,95 \% \mathrm{CI}: 2.73,6.68)$. Individuals in the $5^{\text {th }}$ quintile of any one of the BCAAs

13 also had higher prevalence of these metabolic dysfunction markers, but the risk was lower than

14 for glutamate (see Supplemental Figure 1). The risk of having high blood pressure was not

15 significantly higher in the $5^{\text {th }}$ vs the $1^{\text {st }}$ quintile of glutamate, leucine and isoleucine. It was

16 slightly elevated for the $5^{\text {th }}$ vs the $1^{\text {st }}$ circulating valine quintile (Supplemental Figure 1).

17 Non-genetic effect of circulating amino acids and the link with abdominal obesity

18 We then sought to investigate the contribution of genetic vs. non-genetic factors to the

19 relationship between glutamate and abdominal obesity. As such, we selected $108 \mathrm{MZ}$ twins of

20 the 4665 TwinsUK participants where the co-twins were discordant for trunk fat. The

21 characteristics of these $54 \mathrm{MZ}$ twin pairs are presented in Table 1. Mean intra-pair trunk fat

22 difference was $14.75 \%$ and on average, the heavier co-twin had greater adiposity parameters,

23 triglycerides and HOMA-IR values as well as lower HDL-C levels. 
1 As shown in Figure 2, heavier twins of discordant pairs had significantly higher levels of

2 circulating glutamate, indicating that the relationship between this amino acid and trunk fat is at

3 least partially independent from genetic background. Heavier twins also had higher circulating

4 levels of BCAAs than their leaner co-twins.

6 Next, we attempted to disentangle tissue-specific molecular mechanisms potentially underlying

7 the observed relationship between circulating glutamate and abdominal obesity. To this end, we

8 used a subset of the TwinsUK cohort where SAT samples have been collected for transcriptome

9 profiling as part of the MuTHER study. We applied a linear mixed model linking circulating

10 glutamate with SAT gene expression and identified 224 genes for which mRNA abundance was

11 significantly (adjusted $\mathrm{p}<0.05$ ) associated with circulating glutamate (Supplemental Table 2).

12 Interestingly, among the genes inversely associated with glutamate was GLUL (adjusted

$13 \mathrm{p}=0.0003$, beta=0.089), encoding for Glutamate-Ammonia Ligase, a protein belonging to the

14 glutamine synthetase family and mapping to the Glutamine Biosynthesis I pathway ( $\mathrm{p}=0.0204$,

15 supplemental Table 3). We then tested the direct association between GLUL expression and

16 trunk fat mass $(\%)$ and found a strong negative relationship (beta=-0.03, adjusted $\mathrm{p}=4.4 \mathrm{e}-82$ )

17 which was maintained also after adjusting for BMI (beta=-0.03, adjusted $\mathrm{p}=1.4 \mathrm{e}-38$ ). We also

18 used overlapping data from the similar sized METSIM Study including 800 male samples

19 where VAT samples have been collected for transcriptome profiling (16). In this sample, GLUL

20 expression was negatively associated with both BMI (beta=-0.46, adjusted $\mathrm{p}=3.7 \mathrm{e}-38$ ) and waist-

21 to-hip ratio (beta $=-0.23$, adjusted $\mathrm{p}=1.01 \mathrm{e}-8$ ). 


\section{Adipose tissue transcriptome associated with circulating glutamate in bariatric patients}

2 To further investigate the adipose tissue transcriptome associated with circulating glutamate, we

3 used VAT and SAT biopsy samples from bariatric surgery patients and performed analysis on

4 the SVF and adipocyte fractions. The complete list of genes associated with glutamate, leucine,

5 isoleucine and valine in each cell fraction of each depot is presented in Supplemental Table 5.

6 We did not find a significant association between circulating glutamate and GLUL expression, be

7 it in the SVF or adipocyte and in VAT or SAT. We used IPA to annotate the genes significantly

8 associated with glutamate to canonical pathways. All significant pathways $(\mathrm{p}<0.05)$ are shown in

$9 \quad$ Figure 3.

10 Glutamate signaling across adipose depots and cell populations

11 To further validate the origin of GLUL expression across depots and adipose tissue cell fractions,

12 we accessed untargeted genome-wide differential expression analysis derived from bariatric

13 patients (Supplemental Table 4). We first observed a trend towards higher GLUL expression in

14 SVF vs adipocyte fraction. Restricting to SVF only, we found that GLUL was significantly more

15 expressed in the visceral than the subcutaneous depot $(\log 2$ Foldchange $=0.47, p=0.035)$. We then

16 utilized our recent single-cell adipose tissue expression atlas (17) and were able to validate the

17 more pronounced expression of GLUL in VAT vs SAT specifically in progenitor cells (avg.

$18 \log \mathrm{FC}=0.27$, adjusted $\mathrm{p}=1.74 \mathrm{E}-07)($ Supplemental Figure 2).

19 Finally, in order to explore potentially additional expression signatures related to GLUL

20 signaling, we identified 1,821 genes (Supplemental Table 6) that were upregulated in SVF

21 VAT. Interestingly, pathway analysis on these SVF-VAT genes identified "Glutamate Receptor

22 Signaling" as one of top-three enriched pathways (p-value=2.34e-05, Supplemental Table 7) 
1 including 12 (out of 38) mapped genes DLG4, GLS, GRIK1, GRIK5, GRIN1, GRIN2A, GRIN2C,

2 GRIP1, GRM4, GRM8, HOMER1, SLC38A1 (Figure 4). In our single cell data, 18 out of 38

3 genes mapping to the Glutamate Receptor Signalling pathway were expressed in at least one

4 adipose progenitor/stem cell from either depot. Twelve of these showed differential expression

5 (nominal $\mathrm{p}<0.05$ ) across depots with 9 being upregulated in visceral-derived progenitor cells,

6 including the aforementioned GLUL (Supplemental Table 8). The top visceral-specific genes

7 reaching statistical significance after correction for multiple testing are shown in Supplemental

8 Figure 2. This analysis indicates that expression of many genes related to glutamate are more

9 expressed in VAT compared to SAT and in SVF compared to adipocytes.

\section{DISCUSSION}

11 In this study, we aimed to confirm that circulating glutamate is associated with abdominal

12 obesity. Moreover, we wanted to investigate the adipose tissue transcriptomic profile associated

13 with circulating glutamate to explore potential pathways relevant to this association. In 4,665

14 individuals from the TwinsUK cohort, we report that people with the highest circulating

15 glutamate level have a significantly higher prevalence of abdominal obesity (OR: 7.00, CI: 5.21-

16 9.39) compared to those with the lowest glutamate level. Moreover, analyses on identical twins

17 discordant for abdominal adiposity showed that the positive relationship between plasma

18 glutamate and abdominal fat is at least partially independent of genetic background. In the

19 MuTHER sample, we found that circulating glutamate level was inversely associated with SAT

20 GLUL gene expression. Moreover, we showed that GLUL expression is greater in visceral than

21 subcutaneous adipose tissue.

22 Our observation that glutamate level is significantly associated with abdominal obesity

23 prevalence is concordant with our previous reports that circulating glutamate can correctly 
1 identify $78 \%$ of women presenting visceral obesity assessed by CT scan (18) as well as $90 \%$ of

2 women and men presenting abdominal obesity assessed with WC (19). It is also in line with

3 various reports showing that circulating glutamate is correlated with measurements of central fat

4 accumulation in different populations (3-5). The present study adds to the increasing number of

5 studies now supporting this notion and provides clues regarding it potential bases.

6 The impact of genetics on circulating metabolites was studied by Shin et al. in 2014 (20). They

7 reported that glutamate had a relatively low heritability (25\%), compared for example to the

8 BCAAs $(43 \%, 49 \%$ and $41 \%$ for leucine, isoleucine and valine respectively). This is concordant

9 with our results on MZ twins discordant for abdominal obesity.

10 To our knowledge, this is the first study describing the adipose tissue transcriptome associated

11 with circulating glutamate. The GLUL gene codes for the enzyme Glutamate-Ammonia Ligase,

12 which catalyses the addition of an amino group to glutamate to create glutamine. In 2020, Petrus

13 et al. reported that expression of GLUL in SAT was lower in obese vs lean women (21). This was

14 accompanied by a lower glutamine concentration in adipose tissue and in plasma. Moreover,

15 GLUL expression was normalized (increased) after weight loss by bariatric surgery. In this

16 article, the authors showed that low adipose tissue glutamine concentration was associated with

17 increased expression of genes coding for pro-inflammatory cytokines (CCL2, CD68 et EMR1).

18 Their experiments suggest that this is mediated by an increased glycolysis which in turn

19 increases the O-GlcNAcylation of chromatin and modifies expression of pro-inflammatory

20 genes. In this framework, GLUL expression, abundance and activity could possibly play a causal

21 role in adipose tissue inflammation, which requires further experimentation. If this is the case,

22 and if plasma concentration of glutamate tracks with GLUL, it could potentially be a marker of

23 adipose tissue inflammation. 
1 Some pathways identified in adipose tissue as being associated with circulating glutamate were

2 related to immunity and inflammation: complement system, dendritic cell maturation, death

3 receptor signaling and IL-15 production in SAT as well as interferon signaling in SAT SVF and

4 the inflammasome pathway in VAT SVF. Because excess visceral adiposity correlates with

5 adipose tissue inflammation in both SAT and VAT (22), an association between glutamate and

6 inflammation could potentially explain the association that we and others have reported between

7 circulating glutamate and abdominal/visceral fat accumulation.

8 We identified the Glutamate Receptor Signaling pathway as being upregulated in the SVF of

9 VAT. Studies have shown that VAT is more infiltrated by immune cells, such as macrophages,

10 compared to SAT (22). Moreover, SVF is the adipose tissue cell fraction containing these

11 immune cells (22). Therefore, finding genes related to glutamate metabolism to be most

12 expressed in this depot and cell fraction is concordant with the association we saw in other

13 analyses between circulating glutamate and adipose tissue inflammation genes/pathways. The

14 relevance of this pathway in adipose tissue remains to be established.

15 In all, our results suggest that the association between circulating glutamate and central obesity is

16 related to the expression of GLUL and inflammatory genes in adipose tissue. We acknowledge

17 that GLUL expression was not associated with circulating glutamate in our sample of bariatric

18 patients. This might be due to the smaller sample size or because the association is blunted in

19 patients with severe obesity. Further studies are needed to confirm the association between

20 circulating glutamate and GLUL expression in adipose tissue and whether obesity level

21 influences this association. Moreover, the direction and causality of the associations needs to be

22 elucidated. The strengths of this study include the large number of participants in the TwinsUK-

23 related analysis as well as the use of different adipose tissue depots and cell types in the RNAseq 
1 analyses. One limitation is that the samples for both include mostly Caucasian women, making

2 extrapolation to other population subgroups difficult.

3 In conclusion, we have confirmed in a large cohort that elevated circulating glutamate is

4 associated with a higher prevalence of central obesity. Concomitant alterations in the expression

5 of GLUL and inflammatory genes are observed in adipose tissue, which may provide insight into

6 this association. 


\section{AKNOWLEDGEMENTS}

TwinsUK is funded by the Wellcome Trust, Medical Research Council and the National Institute for Health Research (NIHR)-funded Bioresource, Clinical Research Facility and Biomedical Research Centre based at Guy's and St Thomas' NHS Foundation Trust in partnership with King's College London. The Genotype-Tissue Expression (GTEx) Project was supported by the Common Fund of the Office of the Director of the National Institutes of Health, and by NCI, NHGRI, NHLBI, NIDA, NIMH, and NINDS. The data used for the analyses described in this manuscript were obtained from the dbGaP accession number phs000424.v7.p2 on 07/10/2019. The Authors acknowledge the invaluable collaboration of the surgery team, bariatric surgeons and biobank staff of the IUCPQ as well as Marie-Frederique Gauthier who performed cell fraction isolations in the bariatric samples. AT is co-director of the Research Chair in Bariatric and Metabolic Surgery at Laval University. IMP is the recipient of a doctoral scholarship from the Canadian Institutes of Health Research and received funding from Diabète Québec for these analyses.

\section{DISCLOSURES}

AT received research funding from Johnson \& Johnson Medical Companies, Medtronic, Bodynov and GI Windows for studies on bariatric surgery. 


\section{REFERENCES}

1. Newgard CB. Metabolomics and Metabolic Diseases: Where Do We Stand? Cell metabolism 25: 43-56, 2017.

2. Boulet MM, Chevrier G, Grenier-Larouche T, Pelletier M, Nadeau M, Scarpa J, Prehn C, Marette A, Adamski J, and Tchernof A. Alterations of plasma metabolite profiles related to adipose tissue distribution and cardiometabolic risk. American journal of physiology Endocrinology and metabolism 309: E736-746, 2015.

3. Kimberly WT, O'Sullivan JF, Nath AK, Keyes M, Shi X, Larson MG, Yang Q, Long MT, Vasan R, Peterson RT, Wang TJ, Corey KE, and Gerszten RE. Metabolite profiling identifies anandamide as a biomarker of nonalcoholic steatohepatitis. JCI Insight 2: 2017.

4. Yamakado M, Tanaka T, Nagao K, Ishizaka Y, Mitushima T, Tani M, Toda A, Toda E, Okada M, Miyano H, and Yamamoto H. Plasma amino acid profile is associated with visceral fat accumulation in obese Japanese subjects. Clinical obesity 2: 29-40, 2012.

5. Menni C, Migaud M, Glastonbury CA, Beaumont M, Nikolaou A, Small KS, Brosnan MJ, Mohney RP, Spector TD, and Valdes AM. Metabolomic profiling to dissect the role of visceral fat in cardiometabolic health. Obesity (Silver Spring, Md) 24: 1380-1388, 2016.

6. Moayyeri A, Hammond CJ, Valdes AM, and Spector TD. Cohort Profile: TwinsUK and healthy ageing twin study. International journal of epidemiology 42: 76-85, 2013.

7. Lemieux S, Prud'homme D, Bouchard C, Tremblay A, and Despres JP. A single threshold value of waist girth identifies normal-weight and overweight subjects with excess visceral adipose tissue. The American journal of clinical nutrition 64: 685-693, 1996.

8. Small KS, Hedman AK, Grundberg E, Nica AC, Thorleifsson G, Kong A, Thorsteindottir U, Shin SY, Richards HB, Soranzo N, Ahmadi KR, Lindgren CM, Stefansson K, Dermitzakis ET, Deloukas P, Spector TD, and McCarthy MI. Identification of an imprinted master trans regulator at the KLF14 locus related to multiple metabolic phenotypes. Nature genetics 43: 561-564, 2011.

9. Grundberg E, Small KS, Hedman Å K, Nica AC, Buil A, Keildson S, Bell JT, Yang TP, Meduri E, Barrett A, Nisbett J, Sekowska M, Wilk A, Shin SY, Glass D, Travers M, Min JL, Ring S, Ho K, Thorleifsson G, Kong A, Thorsteindottir U, Ainali C, Dimas AS, Hassanali N, Ingle C, Knowles D, Krestyaninova M, Lowe CE, Di Meglio P, Montgomery SB, Parts L, Potter S, Surdulescu G, Tsaprouni L, Tsoka S, Bataille V, Durbin R, Nestle FO, O'Rahilly S, Soranzo N, Lindgren CM, Zondervan KT, Ahmadi KR, Schadt EE, Stefansson K, Smith GD, McCarthy MI, Deloukas P, Dermitzakis ET, and Spector TD. Mapping cis- and trans-regulatory effects across multiple tissues in twins. Nature genetics 44: 1084-1089, 2012.

10. Menni C, Kastenmuller G, Petersen AK, Bell JT, Psatha M, Tsai PC, Gieger C, Schulz H, Erte I, John S, Brosnan MJ, Wilson SG, Tsaprouni L, Lim EM, Stuckey B, Deloukas P, Mohney R, Suhre K, Spector TD, and Valdes AM. Metabolomic markers reveal novel pathways of ageing and early development in human populations. International journal of epidemiology 42: 1111-1119, 2013. 
11. Tchernof A, Bélanger C, Morisset AS, Richard C, Mailloux J, Laberge P, and Dupont P. Regional differences in adipose tissue metabolism in women: minor effect of obesity and body fat distribution. Diabetes 55: 1353-1360, 2006.

12. Bolger AM, Lohse M, and Usadel B. Trimmomatic: a flexible trimmer for Illumina sequence data. Bioinformatics (Oxford, England) 30: 2114-2120, 2014.

13. Dobin A, Davis CA, Schlesinger F, Drenkow J, Zaleski C, Jha S, Batut P, Chaisson M, and Gingeras TR. STAR: ultrafast universal RNA-seq aligner. Bioinformatics (Oxford, England) 29: 15-21, 2013.

14. Anders S, Pyl PT, and Huber W. HTSeq--a Python framework to work with highthroughput sequencing data. Bioinformatics (Oxford, England) 31: 166-169, 2015.

15. Love MI, Huber W, and Anders S. Moderated estimation of fold change and dispersion for RNA-seq data with DESeq2. Genome biology 15: 550, 2014.

16. Civelek M, Wu Y, Pan C, Raulerson CK, Ko A, He A, Tilford C, Saleem NK, Stančáková A, Scott LJ, Fuchsberger C, Stringham HM, Jackson AU, Narisu N, Chines PS, Small KS, Kuusisto J, Parks BW, Pajukanta P, Kirchgessner T, Collins FS, Gargalovic PS, Boehnke M, Laakso M, Mohlke KL, and Lusis AJ. Genetic Regulation of Adipose Gene Expression and Cardio-Metabolic Traits. American journal of human genetics 100: 428-443, 2017.

17. Vijay J, Gauthier MF, Biswell RL, Louiselle DA, Johnston JJ, Cheung WA, Belden B, Pramatarova A, Biertho L, Gibson M, Simon MM, Djambazian H, Staffa A, Bourque G, Laitinen A, Nystedt J, Vohl MC, Fraser JD, Pastinen T, Tchernof A, and Grundberg E. Single-cell analysis of human adipose tissue identifies depot and disease specific cell types. Nature metabolism 2: 97-109, 2020.

18. Maltais-Payette I, Boulet MM, Prehn C, Adamski J, and Tchernof A. Circulating glutamate concentration as a biomarker of visceral obesity and associated metabolic alterations. Nutrition \& metabolism 15: 78, 2018.

19. Maltais-Payette I, Allam-Ndoul B, Perusse L, Vohl MC, and Tchernof A. Circulating glutamate level as a potential biomarker for abdominal obesity and metabolic risk. Nutrition, metabolism, and cardiovascular diseases : NMCD 29: 1353-1360, 2019.

20. Shin SY, Fauman EB, Petersen AK, Krumsiek J, Santos R, Huang J, Arnold M, Erte I, Forgetta V, Yang TP, Walter K, Menni C, Chen L, Vasquez L, Valdes AM, Hyde CL, Wang V, Ziemek D, Roberts P, Xi L, Grundberg E, Waldenberger M, Richards JB, Mohney RP, Milburn MV, John SL, Trimmer J, Theis FJ, Overington JP, Suhre K, Brosnan MJ, Gieger C, Kastenmuller G, Spector TD, and Soranzo N. An atlas of genetic influences on human blood metabolites. Nature genetics 46: 543-550, 2014.

21. Petrus P, Lecoutre S, Dollet L, Wiel C, Sulen A, Gao H, Tavira B, Laurencikiene J, Rooyackers O, Checa A, Douagi I, Wheelock CE, Arner P, McCarthy M, Bergo MO, Edgar L, Choudhury RP, Aouadi M, Krook A, and Rydén M. Glutamine Links Obesity to Inflammation in Human White Adipose Tissue. Cell metabolism 31: 375-390.e311, 2020.

22. Chait A, and den Hartigh LJ. Adipose Tissue Distribution, Inflammation and Its Metabolic Consequences, Including Diabetes and Cardiovascular Disease. Frontiers in cardiovascular medicine 7: 22, 2020. 
bioRxiv preprint doi: https://doi.org/10.1101/2020.11.19.388678; this version posted August 13, 2021. The copyright holder for this preprint (which was not certified by peer review) is the author/funder. All rights reserved. No reuse allowed without permission.






\section{FIGURES CAPTIONS}

Figure 1. Trunk fat and odds ratios of having abdominal obesity according to circulating amino acid quintiles. Left panels: abdominal fat for each circulating amino acid quintile. The lower, middle and top borders of the boxplot represent the second quantile, median and third quantile, respectively. Right panel: Odds ratios of having abdominal obesity of the second, third, fourth and fifth circulating amino acid quintiles, compared to the first. Error bars represent the $95 \%$ confidence interval. Abdominal obesity was defined as having $37.7 \%$ of fat or more in the trunk region. Odds ratios were obtained for mixed logistic regression models, adjusted for age, sex, metabolomic batch and twin pair clustering.

Figure 2. Difference in circulating amino acids between heavier and leaner twins from monozygotic pairs discordant for trunk fat percentage $(n=108,54$ pairs). Twin pairs were considered discordant if the intra-pair trunk fat difference was greater or equal to the $95^{\text {th }}$ percentile of intra-pair trunk fat percentage difference for monozygotic twins, that is $11.61 \%$ of trunk fat difference. In each discordant pair, we identified the leaner and heavier (in terms of trunk fat) individual. Left panels show the circulating amino acid level of all individuals, according to whether they are the leaner of heavier twin of the pair. The right panels show the circulating amino acid difference between the heavier and leaner twin. P-values are from twotailed and paired Student's t-tests.

Figure 3. Top canonical pathways in adipocytes and SVF from SAT and VAT associated with circulating glutamate. Genes significantly associated with glutamate were identified using a linear model using a p-value threshold of $5 \times 10 \mathrm{e}-5$ and annotated to canonical pathways using Ingenuity Pathway Analysis. SAT: subcutaneous adipose tissue, VAT: visceral adipose tissue, SVF: stroma-vascular fraction. 
Figure 4: Expression levels of glutamate receptor genes show increased expression in SVF derived from VAT. Adipocytes from both SAT and VAT had consistently lower expression in comparison with SVF. 


\section{TABLE}

Table 1. Characteristics of the different samples derived from the TwinsUK cohort.

\begin{tabular}{|lcccc|}
\hline \multirow{2}{*}{ Variables } & Whole sample & \multicolumn{2}{c}{ MZ twins discordant for trunk fat } & \multirow{2}{*}{ MuTHER } \\
\cline { 2 - 4 } & & Leaner & Heavier & \\
\hline Sex (Female : Male) & $4400: 265$ & $53: 1$ & $53: 1$ & $689: 0$ \\
Zygosity (MZ : DZ) & $2247: 2418$ & $54: 0$ & $54: 0$ & $270: 419$ \\
Age (years) & $53.02 \pm 13.87$ & $52.03 \pm 12.89$ & $52.79 \pm 13.17$ & $58.77 \pm 9.20$ \\
BMI (kg/m $\left.{ }^{2}\right)$ & $25.98 \pm 4.74$ & $22.70 \pm 3.69$ & $29.01 \pm 4.82$ & $26.47 \pm 4.74$ \\
Trunk fat $(\%)$ & $35.72 \pm 8.76$ & $27.23 \pm 5.97$ & $41.98 \pm 5.76$ & $38.57 \pm 7.57$ \\
WC $(\mathrm{cm})$ & $80.56 \pm 11.71$ & $77.80 \pm 8.95$ & $86.17 \pm 9.43$ & $77.75 \pm 10.99$ \\
Cholesterol (mmol/L) & $5.54 \pm 1.07$ & $5.23 \pm 0.95$ & $5.65 \pm 1.16$ & $5.56 \pm 1.05$ \\
HDL-C (mmol/L) & $1.83 \pm 0.48$ & $1.99 \pm 0.49$ & $1.72 \pm 0.36$ & $1.82 \pm 0.48$ \\
LDL-C (mmol/L) & $3.23 \pm 0.98$ & $2.88 \pm 0.78$ & $3.35 \pm 1.09$ & $3.24 \pm 0.98$ \\
Triglycerides (mmol/L) & $1.09 \pm 0.62$ & $0.80 \pm 0.25$ & $1.23 \pm 0.69$ & $1.10 \pm 0.58$ \\
SBP (mmHg) & $122.31 \pm 17.29$ & $117.40 \pm 16.77$ & $125.20 \pm 17.11$ & $125.02 \pm 19.33$ \\
DBP (mmHg) & $76.25 \pm 11.07$ & $73.75 \pm 11.87$ & $79.60 \pm 10.62$ & $77.02 \pm 9.75$ \\
HOMA-IR & $1.21(0.67-1.75)$ & $0.63(0.43-0.84)$ & $1.44(0.80-2.08)$ & $1.24(0.68-1.79)$ \\
\hline
\end{tabular}

Values are mean \pm standard deviation, except for HOMA-IR index which is presented as median (interquantile range). MZ: monozygotic, DZ: dizygotic, BMI: body mass index, WC: waist circumference, HDL-C: high-density-lipoprotein cholesterol, LDL-C: low-density-lipoprotein cholesterol, SBP: systolic blood pressure, DBP: diastolic blood pressure, HOMA-IR: homeostasic model assessment of insulin resistance 
a) Glutamate

b) Leucine
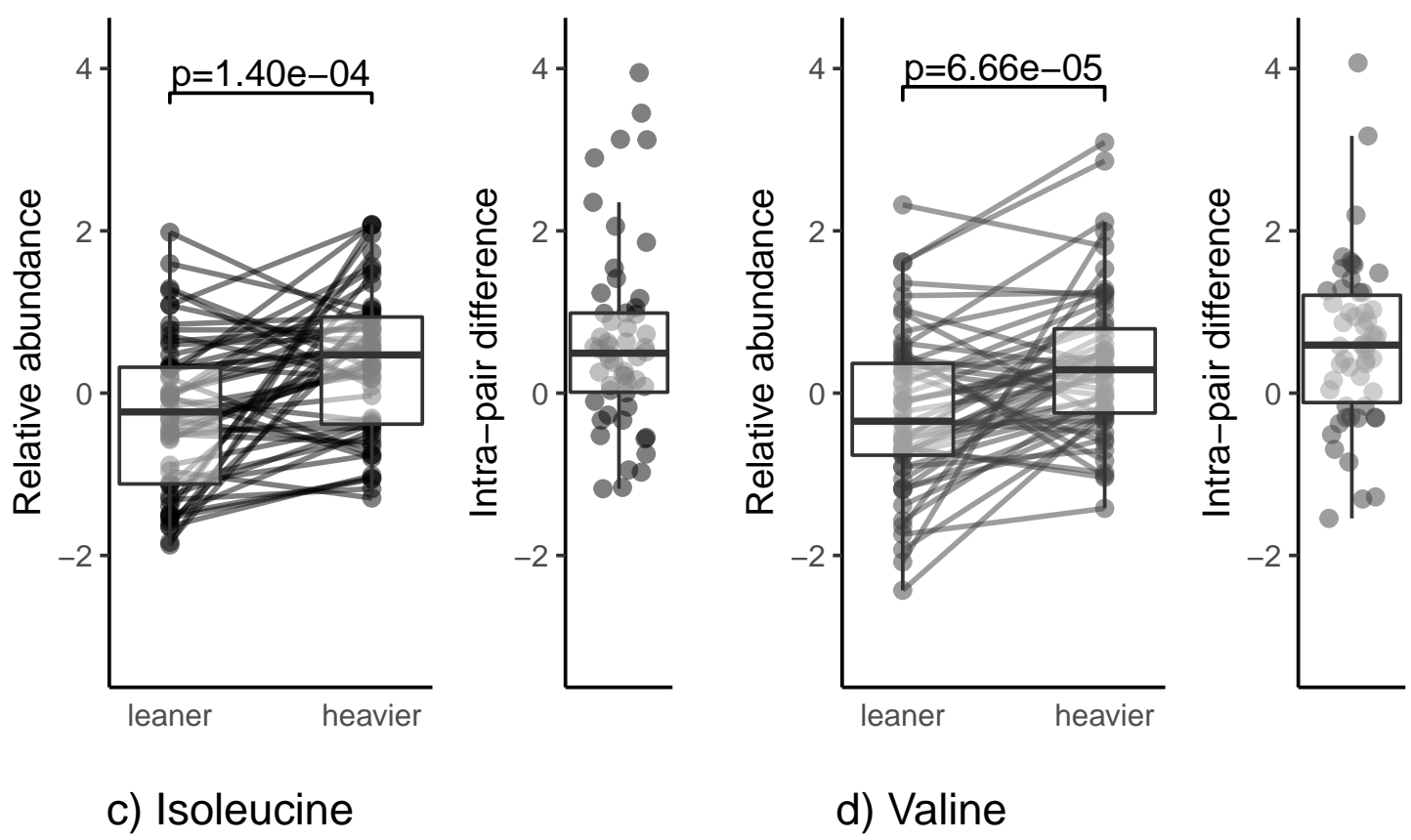

c) Isoleucine
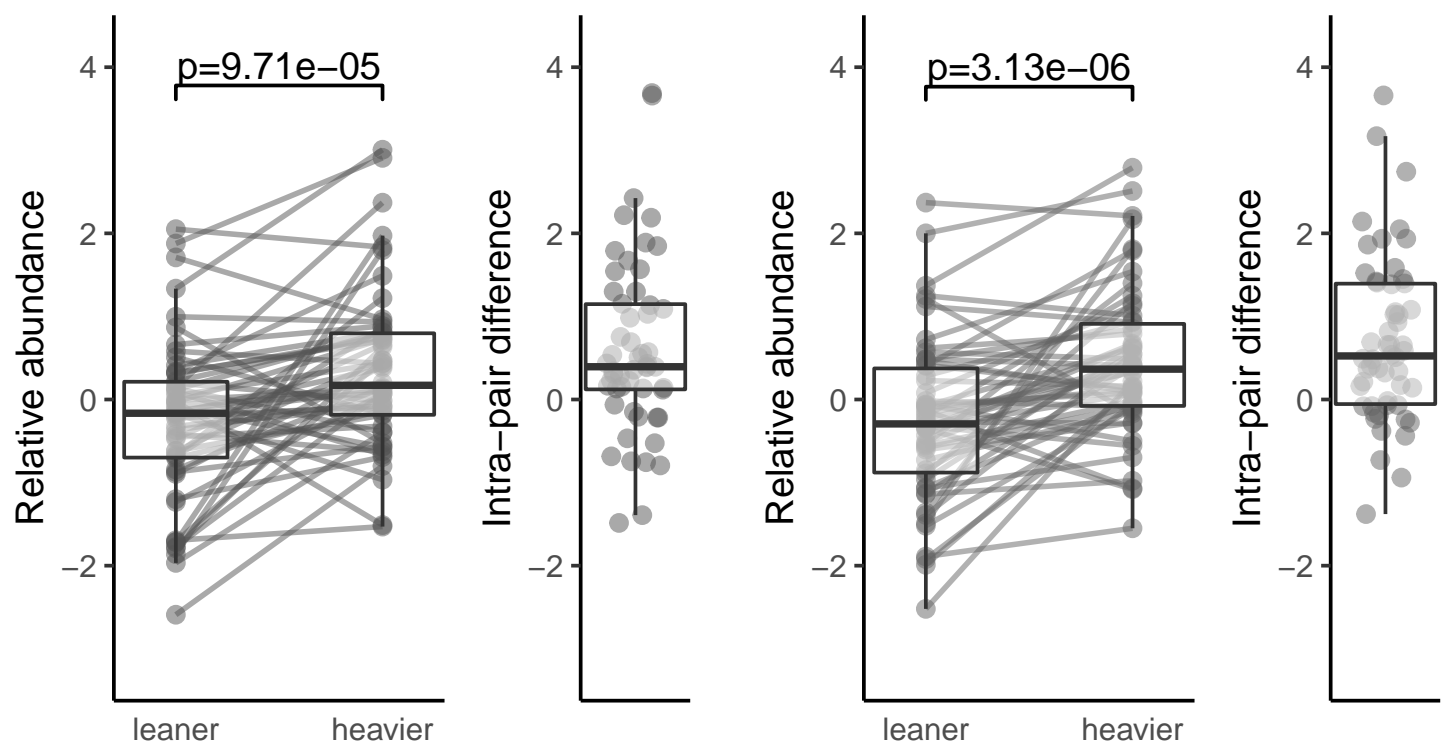
a) SAT - Adipocyte

Nucleotide Excision Repair Pathway

Androgen Signaling

Flavin Biosynthesis IV (Mammalian)

Choline Degradation Assembly of RNA Polymerase II Complex

Anandamide Degradation

Arginine Degradation I (Arginase Pathway)

ERK5 Signaling

Arginine Degradation VI (Arginase 2 Pathway) Urea Cycle

Citrulline Biosynthesis NER Pathway

Axonal Guidance Signaling

Bile Acid Biosynthesis, Neutral Pathway

LXR/RXR Activation

Superpathway of Citrulline Metabolism Human Embryonic Stem Cell Pluripotency

Adipogenesis pathway Methylglyoxal Degradation III

\section{c) VAT - Adipocyte}

Oxidative Phosphorylation

Mitochondrial Dysfunction
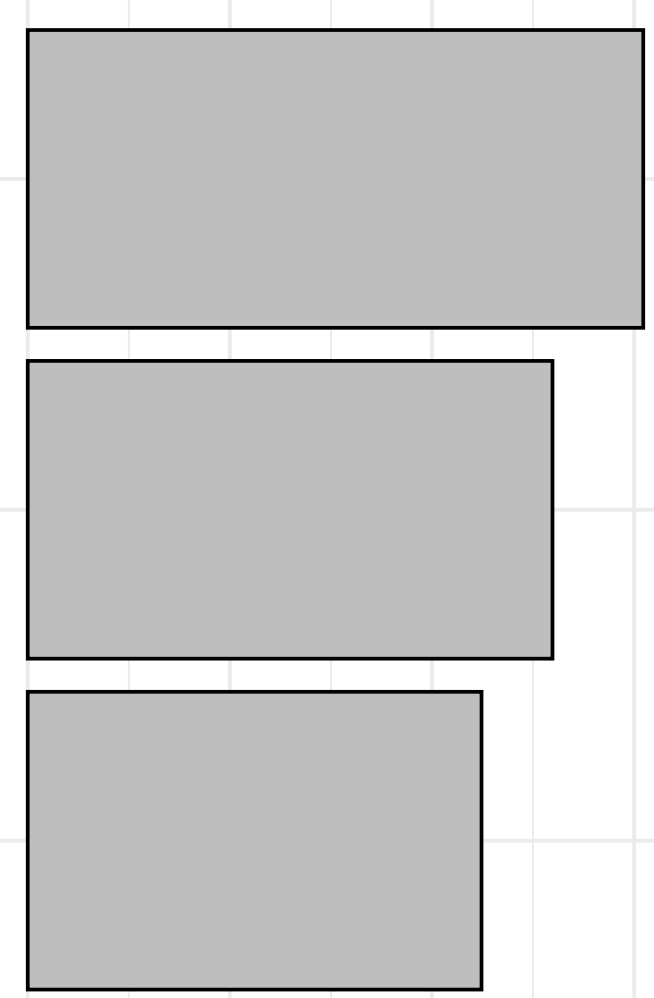

0

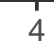

$-\log (p)$
Flavin Biosynthesis IV (Mammalian)

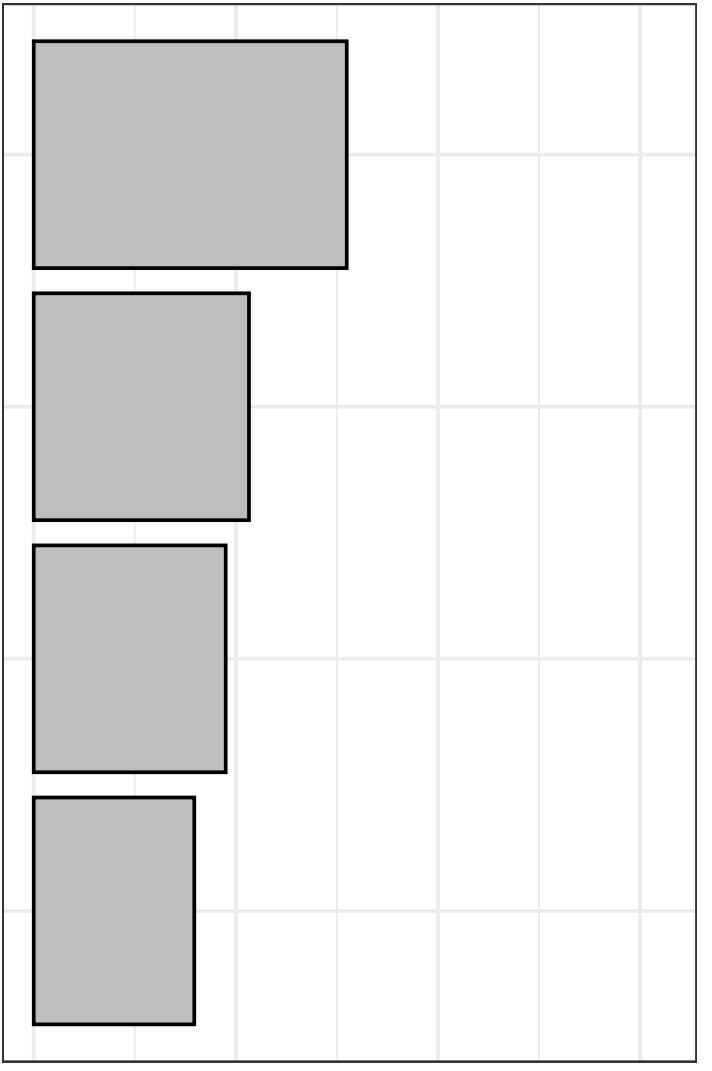

d) VAT - SVF

Endocannabinoid Cancer Inhibition Pathway

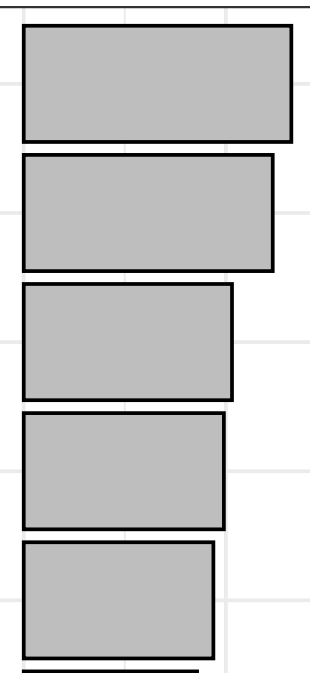

Sphingosine-1-phosphate Signaling

Granzyme B Signaling

Inflammasome pathway
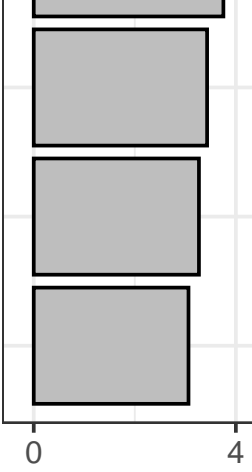

4

$-\log (p)$ 
